\title{
Thickness dependence of the triplet spin-valve effect in superconductor-ferromagnet-ferromagnet heterostructures
}

\author{
Daniel Lenk ${ }^{*}$, Vladimir I. Zdravkov ${ }^{1,2}$, Jan-Michael Kehrle ${ }^{1}$, Günter Obermeier ${ }^{1}$, \\ Aladin Ullrich1, Roman Morari 1,2,3, Hans-Albrecht Krug von Nidda ${ }^{1}$, Claus Müller ${ }^{1}$, \\ Mikhail Yu. Kupriyanov ${ }^{3,4}$, Anatolie S. Sidorenko ${ }^{2}$, Siegfried Horn ${ }^{1}$, Rafael G. Deminov ${ }^{3}$, \\ Lenar R. Tagirov ${ }^{1,3}$ and Reinhard Tidecks ${ }^{1}$
}

Open Access

\author{
Full Research Paper \\ Address: \\ ${ }^{1}$ Institut für Physik, Universität Augsburg, Universitätsstraße 1, \\ D-86159 Augsburg, Germany, ${ }^{2} \mathrm{D}$. Ghitsu Institute of Electronic \\ Engineering and Nanotechnologies ASM, Academiei Str. 3/3, \\ MD2028 Kishinev, Moldova, ${ }^{3}$ Solid State Physics Department, Kazan \\ Federal University, Kremlevskaya Str. 18, 420008 Kazan, Russian \\ Federation and ${ }^{4}$ Skobeltsyn Institute of Nuclear Physics, Moscow \\ State University, Leninskie gory, GSP-1, Moscow 119992, Russia \\ Email: \\ Daniel Lenk* - Daniel.Lenk@physik.uni-augsburg.de \\ * Corresponding author \\ Keywords: \\ heterostructures; superconducting spin valve; thin films; triplet \\ superconductivity
}

Beilstein J. Nanotechnol. 2016, 7, 957-969.

doi:10.3762/bjnano.7.88

Received: 25 February 2016

Accepted: 13 June 2016

Published: 04 July 2016

This article is part of the Thematic Series "Advances in nanomaterials II".

Associate Editor: P. Ziemann

() 2016 Lenk et al.; licensee Beilstein-Institut.

License and terms: see end of document.

\begin{abstract}
Background: In nanoscale layered $\mathrm{S} / \mathrm{F}_{1} / \mathrm{N} / \mathrm{F}_{2} / \mathrm{AF}$ heterostructures, the generation of a long-range, odd-in-frequency spin-projection one triplet component of superconductivity, arising at non-collinear alignment of the magnetizations of $F_{1}$ and $F_{2}$, exhausts the singlet state. This yields the possibility of a global minimum of the superconducting transition temperature $T_{\mathrm{c}}$, i.e., a superconducting triplet spin-valve effect, around mutually perpendicular alignment.
\end{abstract}

Results: The superconducting triplet spin valve is realized with $\mathrm{S}=\mathrm{Nb}$ a singlet superconductor, $\mathrm{F}_{1}=\mathrm{Cu}_{41} \mathrm{Ni}_{59}$ and $\mathrm{F}_{2}=\mathrm{Co}$ ferromagnetic metals, $\mathrm{AF}=\mathrm{CoO}_{x}$ an antiferromagnetic oxide, and $\mathrm{N}=\mathrm{nc}-\mathrm{Nb}$ a normal conducting (nc) non-magnetic metal, which serves to decouple $F_{1}$ and $F_{2}$. The non-collinear alignment of the magnetizations is obtained by applying an external magnetic field parallel to the layers of the heterostructure and exploiting the intrinsic perpendicular easy-axis of the magnetization of the $\mathrm{Cu}_{41} \mathrm{Ni}_{59}$ thin film in conjunction with the exchange bias between $\mathrm{CoO}_{x}$ and $\mathrm{Co}$. The magnetic configurations are confirmed by superconducting quantum interference device (SQUID) magnetic moment measurements. The triplet spin-valve effect has been investigated for different layer thicknesses, $d_{\mathrm{F} 1}$, of $\mathrm{F}_{1}$ and was found to decay with increasing $d_{\mathrm{F} 1}$. The data is described by an empirical model and, moreover, by calculations using the microscopic theory. 
Conclusion: The long-range triplet component of superconducting pairing is generated from the singlet component mainly at the $\mathrm{N} / \mathrm{F}_{2}$ interface, where the amplitude of the singlet component is suppressed exponentially with increasing distance $d_{\mathrm{F} 1}$. The decay length of the empirical model is found to be comparable to twice the electron mean free path of $F_{1}$ and, thus, to the decay length of the singlet component in $\mathrm{F}_{1}$. Moreover, the obtained data is in qualitative agreement with the microscopic theory, which, however, predicts a (not investigated) breakdown of the triplet spin-valve effect for $d_{\mathrm{F} 1}$ smaller than 0.3 to 0.4 times the magnetic coherence length, $\xi_{\mathrm{F} 1}$.

\section{Introduction}

Fulde and Ferrell [1], and Larkin and Ovchinnikov [2] (FFLO) predicted superconductivity on a ferromagnetic background, i.e., in the presence of an exchange field. This was unexpected, because singlet superconductivity is established by pairs of electrons (Cooper pairs) with anti-parallel spin [3], but ferromagnetism leads to a parallel alignment of the electron spins. Indeed, experimental realizations of the FFLO state are scarce [4]. However, a quasi-one-dimensional FFLO-like state can be realized in thin-film superconductor $(\mathrm{S}) /$ ferromagnet $(\mathrm{F})$ proximity-effect systems [5-7].

Here, singlet Cooper pairs in the F-material are formed with zero total spin but non-zero total momentum. This leads to a pairing wave function (PWF), which decays exponentially and oscillates as a function of space [5-7]. As a consequence, interference effects occur, if the PWF is reflected at the outer border of the F-layer of a S/F bilayer, yielding an oscillating superconducting transition temperature as a function of the F-layer thickness, even an extinction of superconductivity with a subsequent recovery (reentrant behavior) is observed [7-9].

Because for two F-layers the superconducting transition temperature depends on the relative orientation of their magnetizations [9-16], trilayers of F/S/F-type and S/F/F-type represent superconducting spin-valves (SSVs) $[10-12,15,16]$. While the $\mathrm{SSV}$ effect in $\mathrm{F} / \mathrm{S} / \mathrm{F}$ structures has been extensively investigated [17-35], this has only been done to a lesser degree in S/F/Ftype structures [36-40]. However, to realize the triplet SSV effect, i.e., an absolute minimum of the superconducting transition temperature, $T_{\mathrm{c}}$, at non-collinear magnetizations [15], the $\mathrm{S} / \mathrm{F} / \mathrm{F}$-type SSV is most suitable, due to the spatial vicinity of the two F-layers [41-47]. Although the dirty-limit theory for the F/S/F-type SSV predicts a contribution of the spin-projection one triplet component to the superconducting $T_{\mathrm{c}}$, a triplet SSV effect is absent $[14,16]$. However, the triplet SSV effect was experimentally realized in F/S/F-type SSVs using elemental ferromagnets $[46,48]$ and has been recently predicted by theory for clean ferromagnets [16].

Without triplet pairing states, the dependence of $T_{\mathrm{c}}$ on the relative magnetic orientation of the F-layers, e.g., in an F/S/F heterostructure, would not be present (at least in the dirty limit).
The reason is that the singlet component does not carry information about the direction of the magnetization, which it is suppressed by [19]. Therefore, the triplet components play a crucial role in S/F proximity-effect devices. In the F-material, pairing states of electrons with opposite spins can be superimposed antisymmetrically to a s-wave singlet and symmetrically to a s-wave triplet spin state with zero spin projection, respectively [49]. Equal-spin pairing yields s-wave triplet pairing states with spin-projection one, which are odd in Matsubara frequency $[6,14,15,49-51]$ like the triplet state with zero spin projection $[49,51]$. Because of the equal spin pairing, the antagonism between the spin ordering of the ferromagnetism and superconductivity is lifted and, thus, the corresponding states are of extraordinarily long range in space and robust against scattering at non-magnetic impurities [49,51] (for the discussion of the decay length see also [52]).

However, only the singlet component enters the self-consistency equation for the superconducting gap and is, thus, the only component directly affecting $T_{\mathrm{c}}[14,15]$. For uniform magnetization of the F-layer in a S/F bilayer and collinear magnetizations in $\mathrm{F} / \mathrm{S} / \mathrm{F}$ and $\mathrm{S} / \mathrm{F} / \mathrm{F}$ trilayers, the singlet and the triplet component with spin-projection zero exist and are coupled with each other $[14,15,49]$. For non-collinear magnetizations, the long-range spin-projection one triplet component is generated, which is coupled to the zero spin-projection triplet and singlet component and, thus, indirectly enters the self-consistency equation [14,15], yielding a change of the superconducting gap and $T_{\mathrm{c}}$. A descriptive picture of the triplet SSV effect is that the generation of the triplet Cooper pairs exhausts the singlet state and, thus, suppresses $T_{\mathrm{c}}$. This yields the possibility of an absolute minimum of the transition temperature as a function of the angle between the magnetizations of $F_{1}$ and $F_{2}$ near the perpendicular orientation [15].

To achieve a non-collinear alignment of the magnetizations of the two F-layers in our $S / F_{1} / F_{2}$-type $S S V$, we exploit the unusual perpendicular easy-axis of magnetization of thin $\mathrm{Cu}_{41} \mathrm{Ni}_{59}$ films $[41,53,54]$ (used as $\mathrm{F}_{1}$ layer material). After cooling the samples in a magnetic field applied parallel to the film plane, the magnetization of the $F_{2}$ layer (a thin Co film) is pinned in the cooling-field direction (in-plane) by the exchange 
bias interaction with a $\mathrm{CoO}_{x}$ film. This shifts the coercive field of the Co film, $H_{\mathrm{c}, \mathrm{Co}}$, to high negative fields with respect to the cooling-field direction, yielding clear separation of the coercive fields of the two ferromagnetic layers [55]. At the small negative coercive field of the $\mathrm{Cu}_{41} \mathrm{Ni}_{59}$ film, $H_{\mathrm{c}, \mathrm{CuNi}}$, the Co film is still in the mono-domain state with in-plane magnetization, while the $\mathrm{Cu}_{41} \mathrm{Ni}_{59}$ film is expected to exhibit a local net magnetization along its easy axis perpendicular to the layer (although the total magnetic moment of the layer is zero).

Thus, with a magnetic field applied parallel to the film plane, we are able to change the magnetic configuration of our $\mathrm{Nb} / \mathrm{Cu}_{41} \mathrm{Ni}_{59} / \mathrm{nc}-\mathrm{Nb} / \mathrm{Co} / \mathrm{CoO}_{x}$ samples (where $\mathrm{nc}-\mathrm{Nb}$ is a normal-conducting spacer, realized by a thin $\mathrm{Nb}$ film below the critical thickness for superconductivity [8,9,56-58], serving to decouple the adjacent ferromagnetic layers) from aligned to crossed, i.e., non-collinear configuration of the two ferromagnetic films.

As the long-range triplet component is predicted to be generated from the singlet component mostly at the interface between the two ferromagnetic layers [15], the strength of the $T_{\mathrm{c}}$ suppression should be strongly dependent on the thickness of the $F_{1}$ layer adjacent to the S-layer, as the singlet PWF decays with increasing distance from the S-layer. The main aim of the present work is to investigate the dependence of the triplet SSV effect on the $F_{1}$ layer thickness. Such investigations are scarce so far $[42,44,47]$.

The generation of spin-triplet Cooper pairs in S/F-heterostructures is expected to be the key to merge superconductivity and spintronics, because spin currents can be realized by supercurrents flowing through ferromagnetic materials, thus minimizing heating effects in spintronic devices [59]. Recently, a triplet SSV was employed to induce magnetism into a normal conducting metal [60]. Triplet SSVs are expected to perform logical functions like the normal conducting giant magnetoresistance (GMR) devices, however, with a much better energy efficiency [61].

\section{Sample preparation and characterization}

The sample series investigated in this work was prepared by magnetron sputtering at room temperature on a commercial silicon substrate with a $\{111\}$ surface. While for the sputtering of the niobium layers, the target was moved over the substrate to obtain a smooth layer of nearly constant thickness, the $\mathrm{Cu}_{41} \mathrm{Ni}_{59}$ layer was deposited on the substrate positioned offaxis below the sputtering target to utilize the natural sputtering gradient of magnetron sputtering to obtain a wedge of varying thickness. Subsequently, the Co layer was deposited on the sub- strate without moving the target. Finally, the $\mathrm{CoO}_{x}$ layer was sputtered from the same target as the metallic Co layer, however, oxygen gas was mixed into the chamber atmosphere to achieve reactive growth of cobalt oxide. For more details on the sputtering procedures see $[8,9,41]$. The heterostructure obtained was then cut perpendicular to the thickness gradient of the $\mathrm{Cu}_{41} \mathrm{Ni}_{59}$ wedge into 25 individual samples of roughly $2.5 \times$ $8 \mathrm{~mm}^{2}$ size, yielding a set of $\mathrm{Nb} / \mathrm{Cu}_{41} \mathrm{Ni}_{59} / \mathrm{nc}-\mathrm{Nb} / \mathrm{Co} / \mathrm{CoO}_{x}$ samples, with varying $\mathrm{Cu}_{41} \mathrm{Ni}_{59}$ layer thickness, which is virtually constant inside a single sample. By preparing all samples within the same run we ensured all sample parameters, which are influenced by the sample preparation (such as interface roughness, boundary resistance, etc.) to be the same for all prepared samples. Figure 1 shows a sketch of the prepared wedge, the dotted lines indicate how individual samples were cut to obtain the sample series.

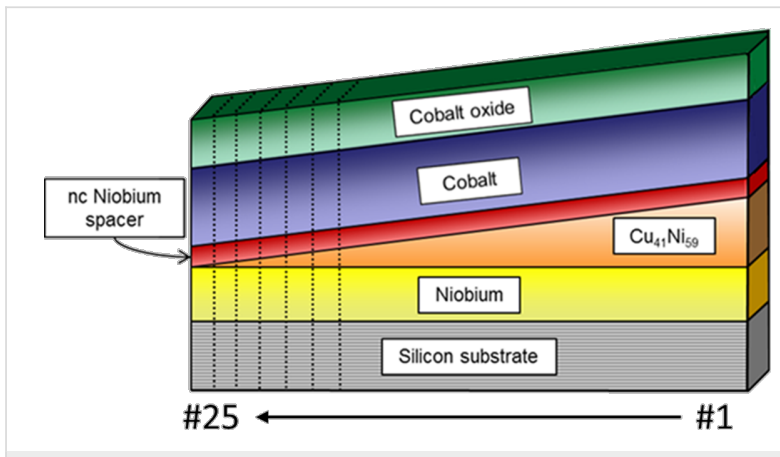

Figure 1: Sketch of the prepared $\mathrm{Nb} / \mathrm{Cu}_{41} \mathrm{Ni}_{59} / \mathrm{nc}-\mathrm{Nb} / \mathrm{Co} / \mathrm{CoO}{ }_{x}$ sample system $\mathrm{SF}_{1} \mathrm{NF}_{2}-\mathrm{AF} 1$. The dotted lines indicate the cutting procedures yielding the individual samples.

We investigated three samples $\left(\mathrm{SF}_{1} \mathrm{NF}_{2}-\mathrm{AF} 1 \# 5\right.$, \#20, and \#25) by cross-sectional transmission electron microscopy (TEM) to check correct deposition and to prove that the interfaces between the layers are clean and smooth. In Figure 2 examples of the obtained images for all three samples are shown. All layers are clearly distinguishable and have sharp and plain interfaces. Furthermore, the layer thicknesses determined by the TEM analysis are used as initial guesses to fit the Rutherford backscattering spectroscopy (RBS) spectra, from which the thicknesses of the layers of further samples are obtained. The thicknesses for the samples investigated by cross-sectional TEM are summarized in Table 1.

About one half of the samples were investigated by RBS analysis. In Figure 3 the results for all constituent layers are shown. The layer thicknesses from the TEM analysis are shown as open symbols. Since the sensitivity of RBS for light elements is small, the adjacent $\mathrm{Co}$ and $\mathrm{CoO}_{x}$ are virtually indistinguishable. Thus, the thickness values for $\mathrm{Co}$ and $\mathrm{CoO}_{x}$ might be slightly different from those given in Figure 3. However, the thick- 


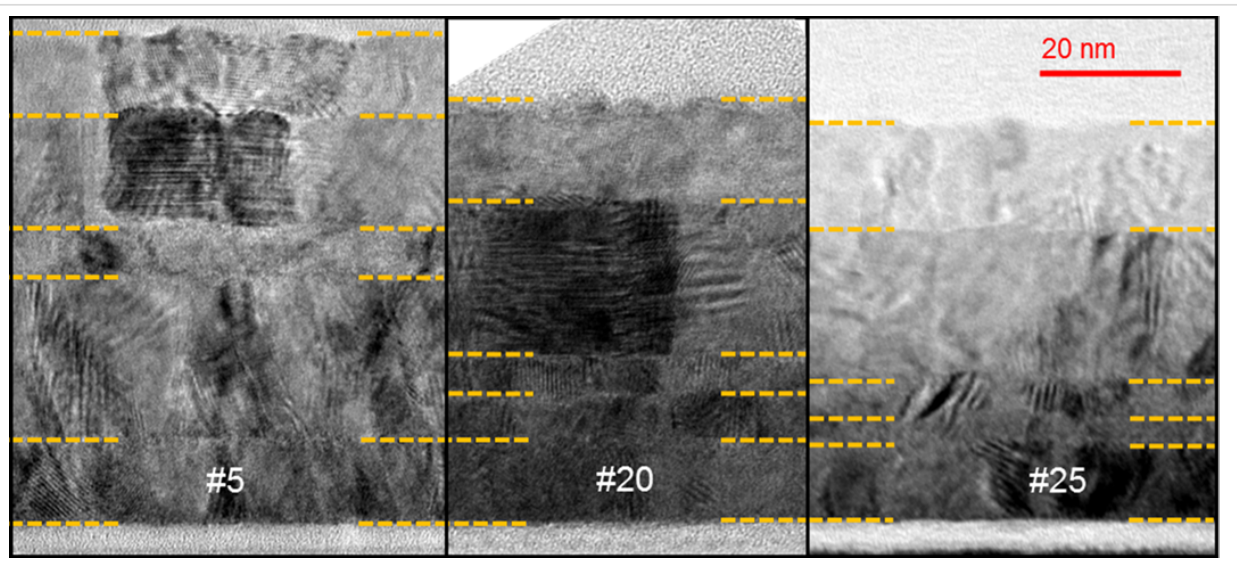

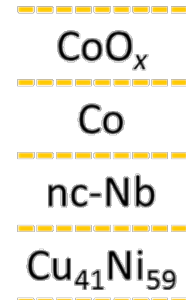

$\mathrm{Nb}$

Figure 2: Cross-sectional TEM images of samples $\mathrm{SF}_{1} \mathrm{NF}_{2}-\mathrm{AF} 1 \# 5$, \#20, and \#25 from left to right. The dashed yellow lines indicate the interfaces between the layers. The obtained layer thicknesses are given in Table 1.

Table 1: Layer thicknesses of several samples of the investigated sample series $\mathrm{SF}_{1} \mathrm{NF}_{2}-\mathrm{AF} 1$ as obtained by a cross-sectional TEM investigation. Please note, that the thicknesses given are evaluated over a larger range than shown in Figure 2.

\begin{tabular}{cccccc} 
sample & \multicolumn{5}{c}{ layer thickness [nm] } \\
& $\mathrm{Nb}$ & $\mathrm{Cu}_{41} \mathrm{Ni}_{59}$ & $\mathrm{nc-Nb}$ & $\mathrm{Co}$ & $\mathrm{CoO}_{x}$ \\
\hline$\# 5$ & 12 & 23 & 7 & 16 & 11.5 \\
$\# 20$ & 12 & 6.5 & 6 & 22 & 14.5 \\
$\# 25$ & 11 & 4 & 5.5 & 22 & 15.5
\end{tabular}

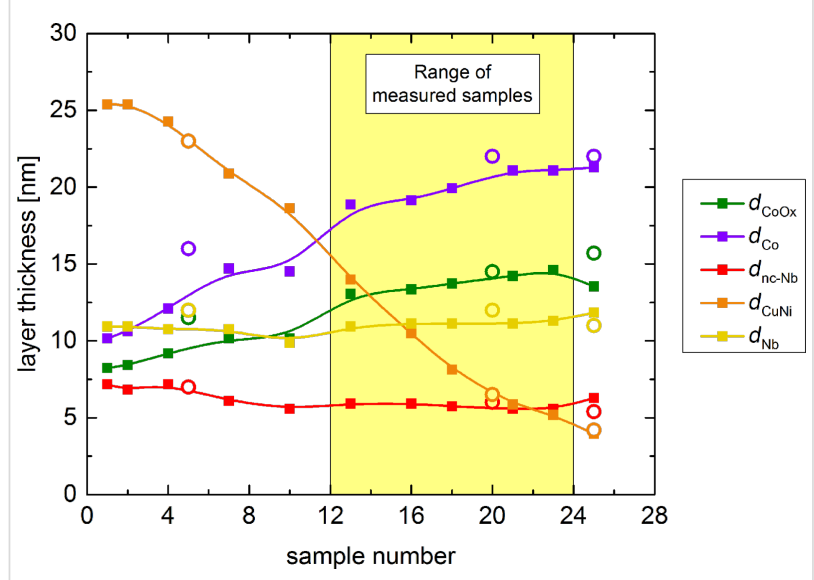

Figure 3: Thickness analysis of the sample series $\mathrm{SF}_{1} \mathrm{NF}_{2}-\mathrm{AF} 1$. While the solid squares show the data obtained by RBS analysis, the open circles are the values from TEM imaging. The solid lines are a guide to the eye.

nesses obtained by TEM investigations and linear interpolations between them were used as initial values for the splitting of the metallic and oxide cobalt signals, cross-checked with the RBS spectra for plausibility and slightly adapted to yield the best fit to the RBS spectra.
Furthermore, we conducted superconducting quantum interference device (SQUID) magnetometry investigations on several $\mathrm{SF}_{1} \mathrm{NF}_{2}-\mathrm{AF} 1$ samples (\#1, \#11, \#13, and \#16) after cooling down to $10 \mathrm{~K}$ in a magnetic field of $10 \mathrm{kOe}$, applied parallel to the film plane and perpendicular to the $\mathrm{Cu}_{41} \mathrm{Ni}_{59}$ gradient, to ensure correct exchange biasing of the Co layer and to determine the field ranges, at which the positive saturated (PS), negative saturated (NS), and crossed (CR) configuration, as well as an antiparallel alignment (APA) of the magnetizations are realized. Exemplary, Figure 4a shows the data for sample $\mathrm{SF}_{1} \mathrm{NF}_{2}-\mathrm{AF} 1 \# 1$. The red arrows indicate the sweep direction of the applied magnetic field. The solid line represents a reconstruction of the hystersis loop based on the simple version of the model of Geiler and co-workers [62].

To further visualize the two contributions to the solid line in Figure $4 \mathrm{a}$, its decomposition into the two constituent ferromagnetic signals is shown in Figure $4 \mathrm{~b}$. For better visibility the $\mathrm{Cu}_{41} \mathrm{Ni}_{59}$ signal was enlarged by a factor of 5 . The corresponding equation is given by

$$
\begin{aligned}
m(H) & =\frac{2}{\pi} m_{\mathrm{s}, \mathrm{Co}} \arctan \left(\frac{H-H_{\mathrm{c}, \mathrm{Co}}}{H_{\mathrm{t}, \mathrm{Co}}}\right) \\
& +\frac{2}{\pi} m_{\mathrm{s}, \mathrm{CuNi}} \arctan \left(\frac{H-H_{\mathrm{c}, \mathrm{CuNi}}}{H_{\mathrm{t}, \mathrm{CuNi}}}\right) \\
& +s H+m_{0},
\end{aligned}
$$

with $m(H)$ being the magnetic moment, $m_{\mathrm{S}}$ the saturation magnetic moment, $H_{\mathrm{c}}$ the coercive field, and $H_{\mathrm{t}}$ a threshold field, determining the field (relative to the coercive field), at which half of the saturation magnetization is realized. The second subscript of the parameters denotes the corresponding material. Moreover, $m_{0}$ is a small offset of the magnetic moment scale 


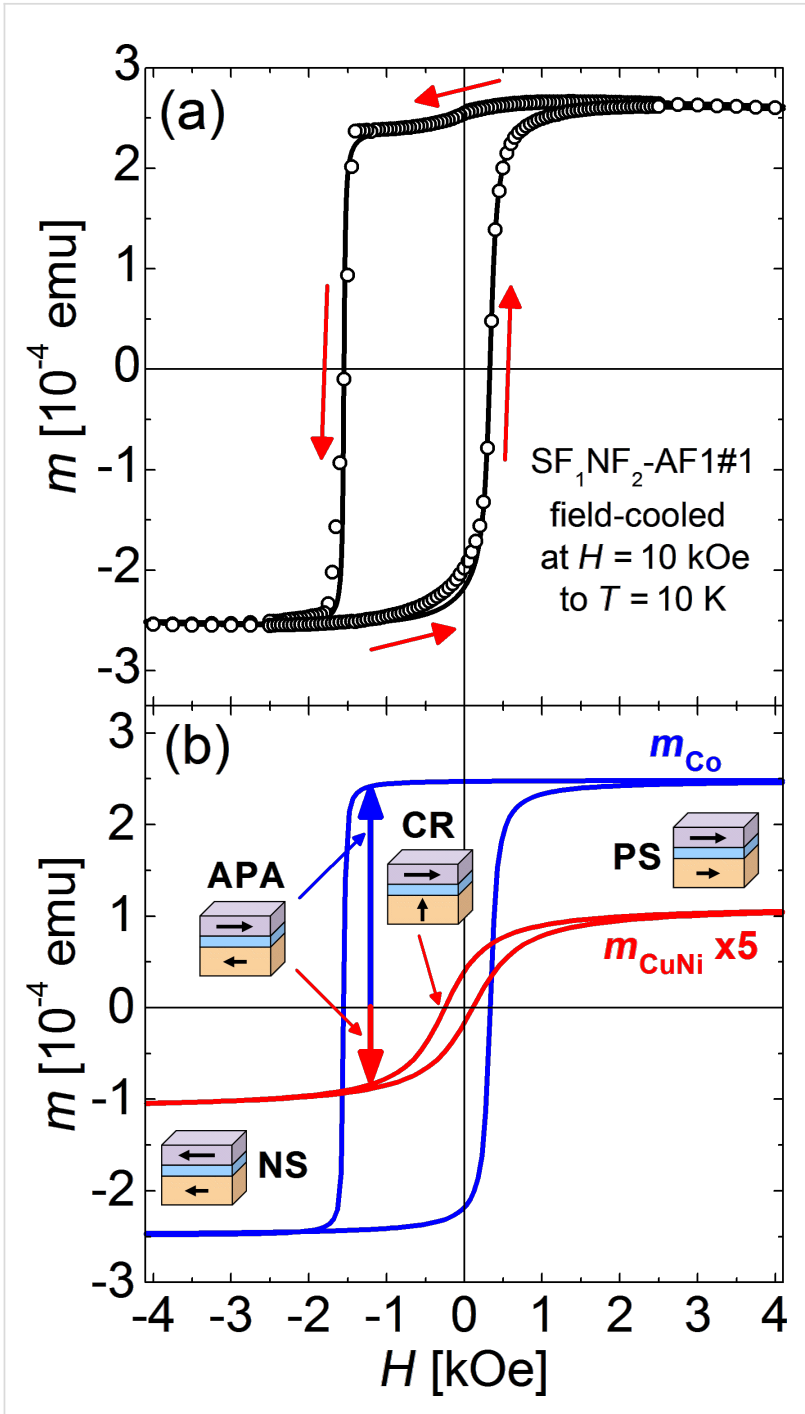

Figure 4: (a) Magnetic hysteresis loop of $\mathrm{SF}_{1} \mathrm{NF}_{2}-\mathrm{AF} 1 \# 1$ recorded by a SQUID magnetometer. Here, $m$ is the magnetic moment and $H$ the magnetic field applied parallel to the film plane. The solid line shows a reconstruction of the hysteresis loop according to the simple version of the model of Geiler and co-workers [62]. The corresponding parameters are summarized in Table 2. The sample was cooled to $10 \mathrm{~K}$ in a field of $10 \mathrm{kOe}$ applied parallel to the film plane. The red arrows indicate the sweep direction. (b) Separation of the obtained hysteresis loop into the constituent loops of the individual layers according to Equation 1. The copper-nickel signal is enlarged by a factor of 5 to improve visibility. The pictograms indicate the different magnetic configurations of the system (PS: positive $(H>0)$ saturated; NS: negative $(H<0)$ saturated; APA: antiparallel alignment of the magnetic moments of the $\mathrm{F}_{1}=\mathrm{Cu}_{41} \mathrm{Ni}_{59}$ and $\mathrm{F}_{2}=\mathrm{Co}$ layers; $\mathrm{CR}$ : crossed configuration of the magnetic moments of $F_{1}$ - and $F_{2}$ layers). The diamagnetic contribution of the silicon substrate is subtracted (only in (b)). Here, cgs emu units are used. In SI units, it is $1 \mathrm{emu}=10^{-3} \mathrm{~A} \cdot \mathrm{m}^{2}$ and $1 \mathrm{Oe}=10^{3} /(4 \pi) \mathrm{A} / \mathrm{m}[63]$, so that (with $\mu_{0}=4 \pi \cdot 10^{-7} \mathrm{~V} \cdot \mathrm{s} /(\mathrm{A} \cdot \mathrm{m})$ ) the magnetic flux density related to $10 \mathrm{kOe}$ is $B=\mu_{0} H=1 \mathrm{~T}$.

and $s$ is the slope of the superposition of the diamagnetic and paramagnetic contribution from the substrate and the antiferromagnet [64], respectively. The parameters are summarized in Table 2. While the $\mathrm{Cu}_{41} \mathrm{Ni}_{59}$ loop is centered at almost zero
Table 2: Parameters used in modeling the hysteresis loop in Figure 4 according to the simple version of the model of Geiler and co-workers [62]. Here, negative and positive sweep direction refers to the semiloops, where the applied field was decreased from positive saturation to negative saturation and vice versa, respectively. Moreover, a linear contribution with a slope of $s=-2.98 \cdot 10^{-6} \mathrm{emu} / \mathrm{kOe}$ and an offset of the magnetic moment scale of $m_{0}=4.4 \cdot 10^{-6}$ emu are considered. For a critical discussion of the used parameters see the text.

\begin{tabular}{ccccc} 
layer & $\begin{array}{c}\text { sweep } \\
\text { direction }\end{array}$ & $m_{\mathrm{s}}\left[10^{-4} \mathrm{emu}\right]$ & $H_{\mathrm{c}}[\mathrm{kOe}]$ & $H_{\mathrm{t}}[\mathrm{kOe}]$ \\
\hline Co & - & 2.49 & -1.551 & 0.015 \\
$\mathrm{Co}$ & + & 2.49 & 0.333 & 0.065 \\
$\mathrm{CuNi}$ & - & 0.22 & -0.251 & 0.400 \\
$\mathrm{CuNi}$ & + & 0.22 & 0.108 & 0.450
\end{tabular}

field, the Co signal is clearly shifted to negative fields by the exchange bias interaction with the $\mathrm{CoO}_{x}$. A similar shift of the Co signal could be detected in all samples, however the weak $\mathrm{Cu}_{41} \mathrm{Ni}_{59}$ contribution is increasingly hard to evaluate, the thinner the $\mathrm{Cu}_{41} \mathrm{Ni}_{59}$ layer is.

We should remark, that the present reconstruction of the hysteresis loop is not unambiguous. Moreover, it shows small deviations from the data, especially for the positive sweep direction around $H=0$. Possibly, these deviations can be reduced by the extended version of the model of Geiler and co-workers [62]. However, this requires the inclusion of three additional fit parameters per layer and sweep direction. This fact, in conjunction with the lack of clear structures in the hysteresis loop, yields mutual dependencies of the parameters and, thus, renders the extended model inapplicable. Moreover, even in the simple version of the model, the obtained parameters include an estimated error of about $\pm 10 \%$ for $m_{S}$ and $\pm(10-20) \%$ for the other parameters.

Investigations of the hysteresis loops of $\left(\mathrm{Cu}_{41} \mathrm{Ni}_{59} / \mathrm{Si}\right) \times 4$ samples show that the magnetically easy axis is directed perpendicular to the film plane [41]. This finding is supported by ferromagnetic resonance (FMR) measurements performed for these samples, as well as by our FMR measurements carried out for single $\mathrm{Cu}_{41} \mathrm{Ni}_{59}$ layers. The result is in agreement with the literature [53].

From the saturation magnetic moment, the magnetic moment per atom can be calculated according to the term $m_{\mathrm{at}} / \mu_{\mathrm{B}}=\left[m_{\mathrm{S}} /\left(V_{\mathrm{L}} N_{\mathrm{A}} / V_{\mathrm{mol}}\right)\right]\left(0.9274 \cdot 10^{-20} \mathrm{emu}\right)^{-1}$. Here, $V_{\mathrm{L}}$ is the volume of the respective layer, $V_{\mathrm{mol}}$ is the molar volume of the respective material, $N_{\mathrm{A}}$ the Avogadro constant and $\mu_{\mathrm{B}}=0.9274 \cdot 10^{-20} \mathrm{emu}[65]$ the Bohr magneton. For $\mathrm{Cu}_{41} \mathrm{Ni}_{59}$ and Co it is $V_{\mathrm{mol}}=6.8 \mathrm{~cm}^{3}$ [9] and $6.62 \mathrm{~cm}^{3}$, respectively. The area of the sample $\mathrm{SF}_{1} \mathrm{NF}_{2}-\mathrm{AF} 1 \# 1$ is $25 \mathrm{~mm}^{2}$. With 
$d_{\mathrm{CuNi}} \approx 25 \mathrm{~nm}$ and $d_{\mathrm{Co}} \approx 10 \mathrm{~nm}$ from Figure 3 and the respective saturation magnetic moments $m_{\mathrm{s}}$ from Table 2, we obtain $m_{\mathrm{at}}=0.0429 \mu_{\mathrm{B}}$ and $m_{\mathrm{at}}=1.18 \mu_{\mathrm{B}}$ for the $\mathrm{Cu}_{41} \mathrm{Ni}_{59}$ and for the Co layer, respectively. The results for $m_{\text {at }}$ are much smaller than the bulk material values of $1.7 \mu_{\mathrm{B}}[66]$ and $0.14 \mu_{\mathrm{B}}[67]$ for Co and $\mathrm{Cu}_{41} \mathrm{Ni}_{59}$, respectively, especially in the latter case.

For isolated $\mathrm{Cu}_{41} \mathrm{Ni}_{59}$ layers with $d_{\mathrm{F}}=21 \mathrm{~nm}$ and $d_{\mathrm{F}}=48 \mathrm{~nm}$, investigated in our previous work [9], which were deposited directly on Si substrates by the same preparation methods used in the present work, $m_{\text {at }}$ determined from $m_{\mathrm{S}}$ shows no reduction from the bulk material value. However, for $\mathrm{Nb} / \mathrm{Cu}_{41} \mathrm{Ni}_{59}$ bilayers with $d_{\mathrm{Nb}}=14.1,13.0$ and $14.2 \mathrm{~nm}$ and $d_{\mathrm{CuNi}}=34.3$, 26.5 , and $18.8 \mathrm{~nm}$, respectively, hysteresis curves measured by SQUID magnetometry at $T=10 \mathrm{~K}$, where the sample is normal conducting, yield $m_{\mathrm{s}}$ values resulting in $m_{\mathrm{at}}=0.074 \mu_{\mathrm{B}}$, $0.080 \mu_{\mathrm{B}}$, and $0.127 \mu_{\mathrm{B}}$.

Investigations of the magnetic properties of thin films of $\mathrm{Co}$ (deposited on W) [68] and $\mathrm{Cu}_{40} \mathrm{Ni}_{60}$ (deposited as $\mathrm{Cu}_{40} \mathrm{Ni}_{60} / \mathrm{Cu}$ multilayers) [53], show that in both cases the magnetization is given by the bulk material value, so that the magnetic moment (for fixed area of the sample) increases linearly with the thickness of the magnetic layer (see Figure 1 and Equation 12 of [68] and Figure 5 and Equation 6 of [53]). However, a non-magnetic "dead" layer of thickness $0.18 \mathrm{~nm}$ and $2 \times 1 \mathrm{~nm}$ at the interfaces to the $\mathrm{W}$ and $\mathrm{Cu}$, respectively, is observed.

Such non-magnetic layers can in principle exist in the $\mathrm{Nb} / \mathrm{Cu}_{41} \mathrm{Ni}_{59}$ bilayers (and also in the sample of the present work, where the $\mathrm{Cu}_{41} \mathrm{Ni}_{59}$ layer is sandwiched between two $\mathrm{Nb}$ layers). While the solubility of $\mathrm{Nb}$ in $\mathrm{Cu}$ is extremely small under ambient conditions, the $\mathrm{Nb}-\mathrm{Ni}$ phase diagram shows a certain solubility of $\mathrm{Nb}$ in $\mathrm{Ni}$ and vice versa [69]. In this case the bilayer with the thinnest $\mathrm{Cu}_{41} \mathrm{Ni}_{59}$ layer would show the largest reduction of $m_{\mathrm{at}}$. However, the opposite is observed.

There may be several sources of experimental error in the absolute value of small magnetic moments present in SQUID measurements [70,71]. However, in the present study these effects should reduce $m_{\mathrm{S}}$ of both magnetic layers by the same factor, yielding the ratio between the saturation magnetizations being unaffected. With $m_{\mathrm{s}}=m_{\mathrm{at}}\left(V_{\mathrm{L}} / V_{\mathrm{mol}}\right) N_{\mathrm{A}}$, where $V_{\mathrm{L}}=A \cdot d_{\mathrm{L}}$ with $A$ and $d_{\mathrm{L}}$ the area of the sample and the thickness of the respective layer, using $m_{\mathrm{at}}=1.7 \mu_{\mathrm{B}}$ and $0.14 \mu_{\mathrm{B}}$ for Co and $\mathrm{Cu}_{41} \mathrm{Ni}_{59}$, respectively, we obtain $m_{\mathrm{s}, \mathrm{Co}} / m_{\mathrm{s}, \mathrm{CuNi}}=$ $12.5\left(d_{\mathrm{Co}} / d_{\mathrm{CuNi}}\right)$. With $d_{\mathrm{Co}} \approx 10 \mathrm{~nm}$ and $d_{\mathrm{CuNi}} \approx 25 \mathrm{~nm}$, we obtain a ratio of 5 . Even if we take into account that the thickness of the Co layer, determined from TEM investigations on $\mathrm{SF}_{1} \mathrm{NF}_{2}-\mathrm{AF} 1 \# 5$, possibly indicates a smaller slope of the thickness profile for small sample numbers than evaluated from RBS (see Figure 3) and assume $d_{\mathrm{Co}} \approx 15 \mathrm{~nm}$ for $\mathrm{SF}_{1} \mathrm{NF}_{2}-\mathrm{AF} 1 \# 1$, the ratio increases only to 7.5 . This is much smaller than the ratio of $2.49 / 0.22=11.3$ obtained from the saturation magnetizations given in Table 2 .

Thus, while the reduced values of $m_{\text {at }}$ observed for S/F bilayers discussed above, might be explained by a reduced value of $m_{\mathrm{S}}$ caused by experimental errors in the SQUID signal, the extraordinary small value of $m_{\text {at }}$ of the $\mathrm{Cu}_{41} \mathrm{Ni}_{59}$ layer in the present work cannot be explained due to such effects only. Therefore, for this layer other mechanisms of reduction of $m_{\mathrm{s}}$ have to be discussed. It has to be considered, that during deposition of the samples in the present work oxygen gas was mixed into the atmosphere of the sputtering chamber during growth of $\mathrm{CoO}_{x}$. This can locally lead to the formation of antiferromagnetic $\mathrm{NiO}$ [65] in the already deposited $\mathrm{Cu}_{41} \mathrm{Ni}_{59}$ layer, yielding a reduction of the magnetic moment of the film. A degradation of $\mathrm{Cu}_{41} \mathrm{Ni}_{59}$ by oxygen has been discussed in our former work on AF-F/S/F and $\mathrm{F} / \mathrm{S} / \mathrm{F}-\mathrm{AF}$ samples, where $\mathrm{F}=\mathrm{Cu}_{41} \mathrm{Ni}_{59}, \mathrm{~S}=\mathrm{Nb}$, and $\mathrm{AF}=\mathrm{CoO}_{x}[72]$. The samples were produced by the same deposition method as in the present work, except that the substrate was heated up to $300{ }^{\circ} \mathrm{C}$ and $200{ }^{\circ} \mathrm{C}$ to deposit the AF bottom and top layer, respectively. In these investigations, for AF-F/S/F samples aging effects (possibly due to oxygen diffusion from the $\mathrm{CoO}_{x}$ ) are observed. The theoretical fitting of the experimental data (parameters given in Table I of [72]) yields an increase of the magnetic coherence length, $\xi_{\mathrm{F} 0}$ (for a definition, see $[7,9]$ ) in the F-layers (corresponding to $\xi_{\mathrm{F} 1}$ in the present work), which is probably indicating a decrease of the exchange energy, $E_{\mathrm{ex}}$. The reason for such a decrease of $E_{\mathrm{ex}}$ can be a weakening of the exchange interaction caused by the formation of NiO. An increase in $\xi_{\mathrm{F} 0}$ is also observed in the F/S/F-AF sample series (see Table I of [72]). However, this series is investigated just after deposition. A possible source of oxygen in this case may be the $\mathrm{CoO}_{x}$ layer deposition after deposition of the $\mathrm{Cu}_{41} \mathrm{Ni}_{59}$ layers. This is the same case in the present study, however, the $\mathrm{CoO}_{x}$ layer is deposited at room temperature here.

\section{Results and Discussion}

To investigate the triplet SSV effect, resistance-temperature $(R(T))$ measurements of the superconducting transition were recorded at different magnetic fields applied parallel to the film plane. The measurements were performed in an Oxford Instruments Heliox sorption-pumped ${ }^{3} \mathrm{He}$ insert, applying the DC four-probe method for both polarities of the sensing current $(10 \mu \mathrm{A})$ to eliminate thermoelectric voltages. The samples were cooled down to liquid helium temperature in a field of $30 \mathrm{kOe}$, applied in the same direction as in the preceding SQUID measurements. By sweeping the temperature at various fixed magnetic fields, the magnetic field dependence of the transition tem- 
perature, $T_{\mathrm{c}}$, was obtained for samples $\mathrm{SF}_{1} \mathrm{NF}_{2}-\mathrm{AF} 1 \# 12$, \#17, \#19, \#22, \#24.

Figure 5 exemplarily shows a plot of the transition curves, obtained for sample $\mathrm{SF}_{1} \mathrm{NF}_{2}-\mathrm{AF} 1 \# 17$. The solid lines represent certain resistance levels. The bold line is the resistance level, at which we evaluated the transition temperature, $T_{\mathrm{c}}$, at one half of the normal state resistance. There are no obvious peculiarities in the shape of the transition curves, even at the coercive field of $\mathrm{Cu}_{41} \mathrm{Ni}_{59}$. At this field the transition is shifted towards lower temperatures over the whole width of the transition.

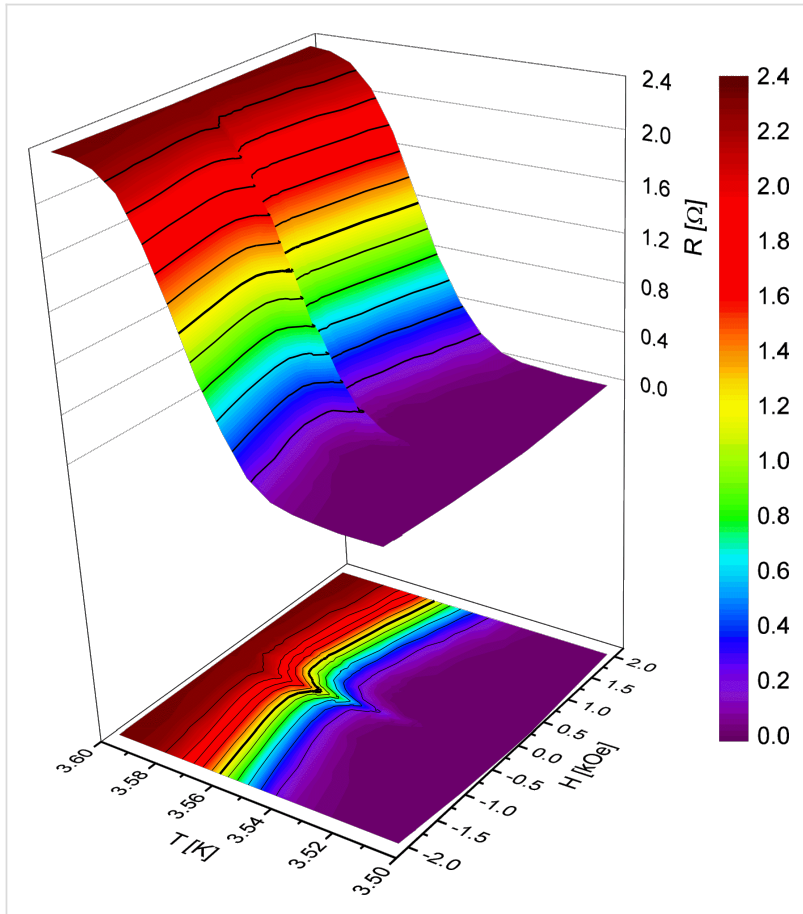

Figure 5: Superconducting transition as a function of the temperature, $T$, and the applied magnetic field, $H$, parallel to the film plane, obtained by four-probe measurements on sample $\mathrm{SF}_{1} \mathrm{NF}_{2}-\mathrm{AF} 1 \# 17$ at a measuring current of $10 \mu \mathrm{A}$, both as $3 \mathrm{D}$ plot and planar projection. The applied magnetic field was varied from positive to negative values. The solid lines represent certain resistance levels, the bold line indicates roughly the level, where we evaluated the transition temperature, $T_{\mathrm{c}}$, at half of the normal-state resistance.

Figure 6a shows the $T_{\mathrm{c}}$ data obtained for sample $\mathrm{SF}_{1} \mathrm{NF}_{2}-$ AF1\#22 as a function of the magnetic field. We observed a narrow and pronounced reduction of $T_{\mathrm{c}}$ at the coercive field of the $\mathrm{Cu}_{41} \mathrm{Ni}_{59}$ layer, which is already visible in the transition curves in Figure 5. We attribute this reduction to the theoretically predicted [15] reduction of the critical temperature by the generation of the long-range, odd-in-frequency, spin-projection one triplet component of superconductivity at non-collinear orientation of the magnetizations of the two ferromagnetic layers, $F_{1}$ and $F_{2}$, as discussed in detail in [41] and outlined in the Introduction section.

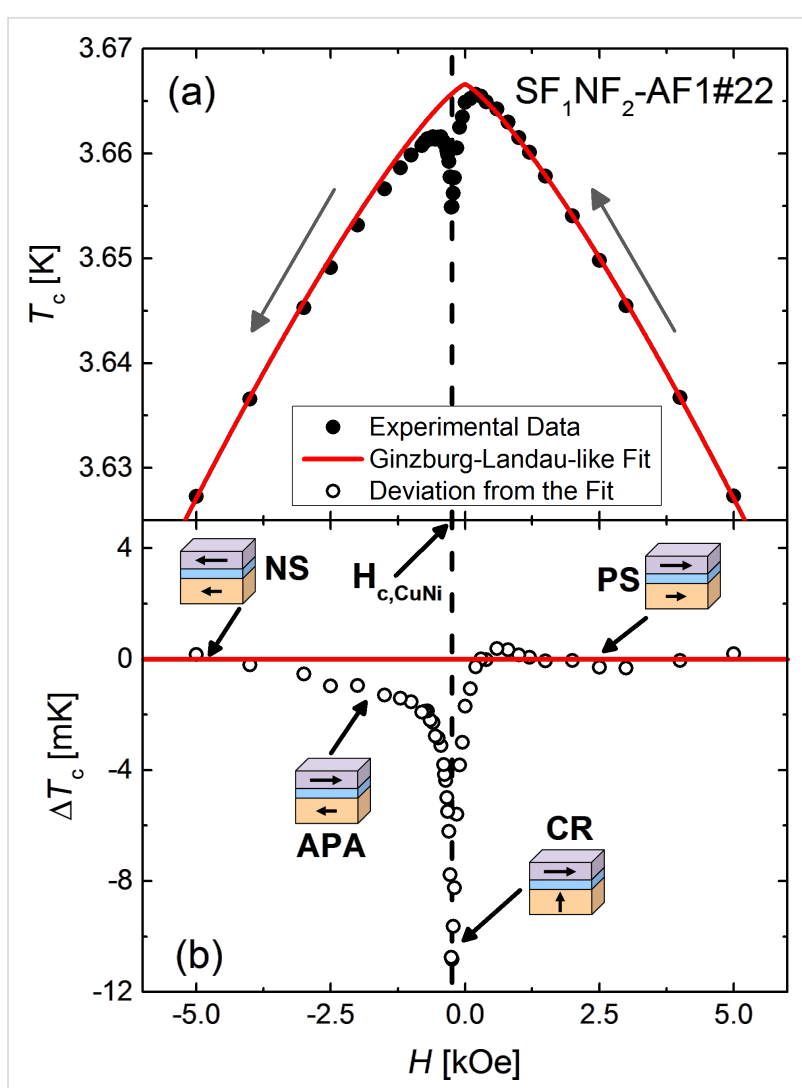

Figure 6: (a) Superconducting transition temperature $T_{\mathrm{C}}$ of $\mathrm{SF}_{1} \mathrm{NF}_{2^{-}}$ AF1\#22 as a function of the applied magnetic field $H$. The direction of the variation of the applied field is given by arrows, the solid red line shows a fit to a Ginzburg-Landau-like behavior (for details see the text). (b) Deviation, $\Delta T_{\mathrm{c}}$, calculated between the experimental data and the fit. The pictograms show the magnetic configurations of the system in accordance with the magnetic measurements in Figure 4. The maximal suppression at crossed configuration of the magnetic layers is evaluated as $\Delta T_{\mathrm{c} \text {, max }}$. Here, $H_{\mathrm{c}, \mathrm{CuNi}}$ is the coercive field of the $\mathrm{Cu}_{41} \mathrm{Ni}_{59}$ alloy layer.

In Chapter III of [41], there is a detailed discussion excluding other possible origins of the observed phenomenon, e.g., stray fields generated by the multi-domain state of the $\mathrm{Cu}_{41} \mathrm{Ni}_{59}$ film at its coercive field. Moreover, recent investigations of a $\mathrm{Nb} / \mathrm{Cu}_{41} \mathrm{Ni}_{59}$ bilayer show no evidence of a reduction of $T_{\mathrm{c}}$ neither at the parallel, nor at the perpendicular applied coercive field (see the comment at the end of Chapter IV.B.2 of [73]).

This behavior is representative for all investigated samples, however, the $T_{\mathrm{c}}$ reduction is varying in magnitude. To separate the triplet SSV effect from the reduction of $T_{\mathrm{c}}$ by the applied magnetic field, we approximate the temperature dependence of the parallel critical field by a Ginzburg-Landau (GL)-like behavior. According to [74], a superconducting thin film shows a temperature dependence of the upper critical field (parallel to the film plane) given by $H_{\mathrm{c}}(T)=H_{\mathrm{c}}(0)\left(1-T / T_{\mathrm{c} 0}\right)^{\alpha}$. Here, $T_{\mathrm{c} 0}$ is the critical temperature, i.e., the superconducting transition temperature in the absence of currents and magnetic fields, and $\alpha$ is 
a parameter given by the effective dimensionality of the superconductor. We should remark, that strictly obeying the definition of $T_{\mathrm{c} 0}$, it can not be defined for the heterostructures of the present work, because magnetic material is present. Here it is used to identify the transition temperature in zero external magnetic field. For a two-dimensional and a three-dimensional superconductor, $\alpha_{2 \mathrm{D}}=1 / 2$ and $\alpha_{3 \mathrm{D}}=1$, respectively. Identifying $T$ with $T_{\mathrm{c}}$ and $H_{\mathrm{c}}$ with $H$ (because we determine the transition temperature, $T_{\mathrm{c}}$ at fixed field $H$ ), we fitted a GL-like behavior given by

$$
\frac{T_{\mathrm{c}}}{T_{\mathrm{c} 0}}=1-\left(\frac{H}{H_{\mathrm{c}}(0)}\right)^{\frac{1}{\alpha}}
$$

to the data points outside the area of reduced $T_{\mathrm{c}}$ and, thus, to data points corresponding to a parallel alignment of the magnetizations of the two ferromagnetic layers. Here, $\alpha$ and $H_{\mathrm{c}}(0)$ and $T_{\mathrm{c} 0}$ are fitting parameters, their dependencies on $d_{\mathrm{CuNi}}$ are given in Figure $7 \mathrm{a}$ and Figure $7 \mathrm{~b}$, and Figure $8 \mathrm{a}$, respectively. All three parameters decrease as a function of $d_{\mathrm{CuNi}}$.

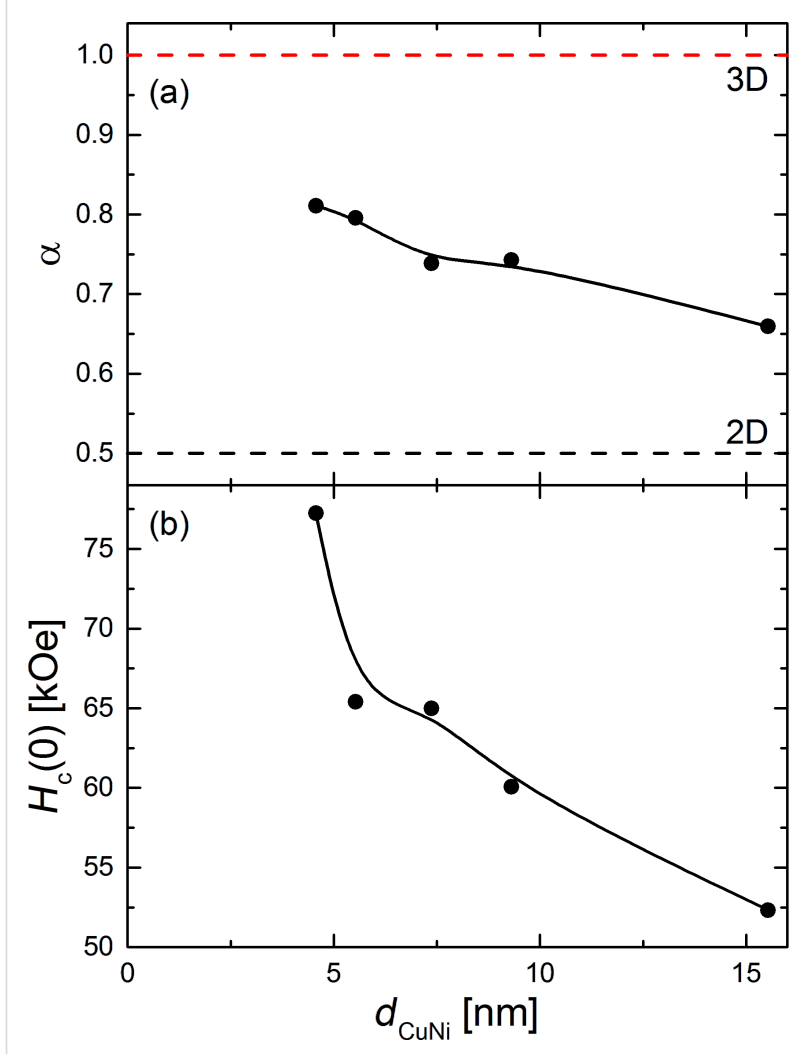

Figure 7: (a) Dimensionality parameter $\alpha$ and (b) the (fictive) upper critical field at zero temperature, $H_{\mathrm{c}}(0)$, as obtained by fitting Equation 2 to the experimental data, as a function of the thickness of the $\mathrm{Cu}_{41} \mathrm{Ni}_{59}$ layer. The solid lines are guide to the eye, the dashed ones in (a) indicate the values of $\alpha$ for a two and a three-dimensional superconductor according to the GL-theory, respectively.

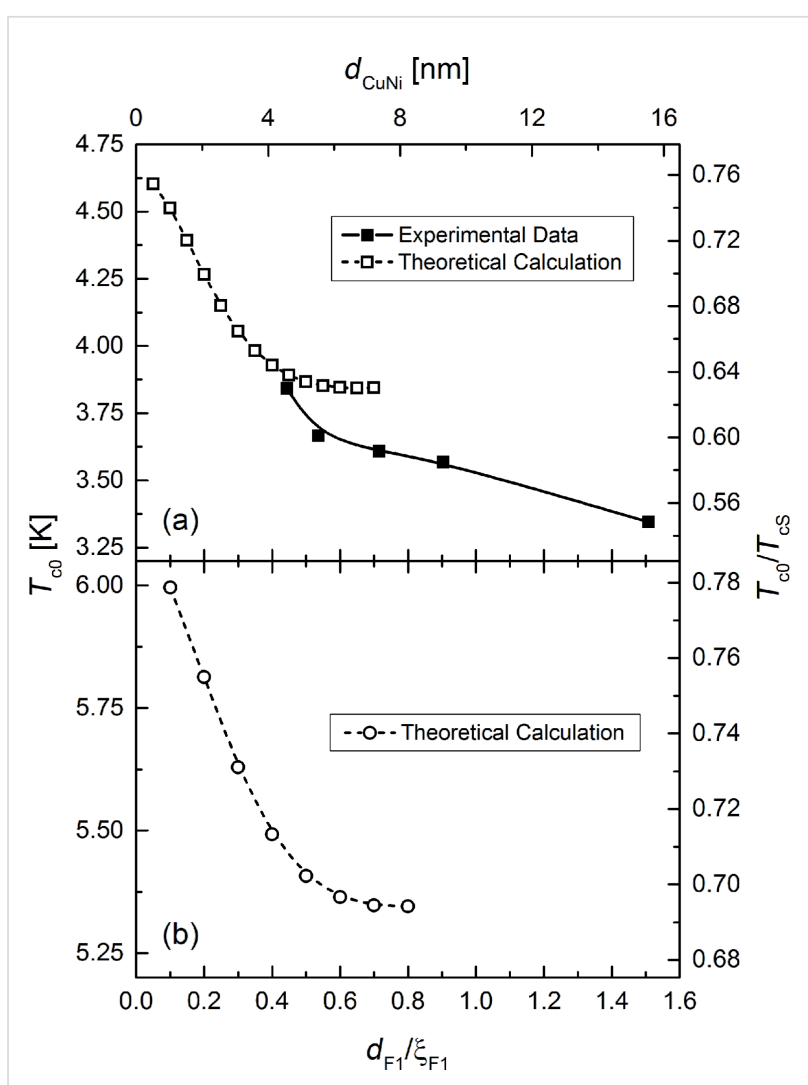

Figure 8: Superconducting transition temperature, $T_{\mathrm{c} 0}$, in zero external magnetic field as a function of the $\mathrm{F}_{1}=\mathrm{Cu}_{41} \mathrm{Ni}_{59}$ layer thickness $d_{\mathrm{F} 1}=d_{\mathrm{CuNi}}$. While the solid squares in (a) show experimental results, the open squares and circles in (a) and (b), respectively, show predictions, which can be derived from the theory of Fominov and co-workers [15]. The solid and dashed lines are guide to the eye. The theoretical data are plotted both in absolute and normalized (with respect to $T_{\mathrm{CS}}$ ) units. In (a), we assumed $T_{\mathrm{cS}}=6.1 \mathrm{~K}$, in (b) we used $T_{\mathrm{cS}}=7.7 \mathrm{~K}$, which is the critical temperature of a free standing $\mathrm{Nb}$ film of $12 \mathrm{~nm}$ thickness. Moreover, we used $\xi_{\mathrm{F} 1}=10.3 \mathrm{~nm}$ for the magnetic coherence length in the $\mathrm{F}_{1}$ layer ( $\mathrm{Cu}_{41} \mathrm{Ni}_{59}$ layer) to plot the data as a function of the reduced thickness $d_{F 1} / \xi_{F 1}$. For a detailed discussion of the material and modeling parameters used for the theoretical calculations, see the Appendix.

For $T_{\mathrm{c} 0}$ this can be theoretically derived in this regime from the theory of Fominov et al. [15] (see Figure 8 for the calculated data, both in absolute units and normalized to $T_{\mathrm{cS}}$, the critical temperature of a freestanding $\mathrm{Nb}$ film of the same thickness as in the present sample series). Since $T_{\mathrm{c} 0}$ decreases, this is also expected for $H_{\mathrm{c}}(0)$. For the S-layer (for which usually $l \ll \xi_{0}$ [73]), we obtain in the twodimensional case $H_{\mathrm{c}}(0) \propto \xi_{0}^{-1 / 2} \propto T_{c 0}^{1 / 2}$ (see Equation 3 of [73]). Here, $l$ is the electron mean free path and $\xi_{0}$ the Bardeen-Cooper-Schrieffer (BCS) coherence length [74]. We do not expect that this is qualitatively different in the present samples.

However, the reason for the decrease of $\alpha$ is not so obvious. This decrease means that the effective dimensionality becomes 
more and more two-dimensional for an increasing thickness of the system, which is counter-intuitive. We can estimate for the S-layer, using typical parameters for our $\mathrm{Nb}$ films $[9,73]$, that the GL coherence length is much larger than $d_{\mathrm{S}}$ in the range for which Equation 2 is applied. Thus, at least the S-layer is estimated to be in the two-dimensional limit.

The difference between the GL-like fit and the experimental data yields the net reduction of the transition temperature, $\Delta T_{\mathrm{c}}$, without the pair breaking by the applied field. In the antiparallel relative alignment (APA) of the magnetization in the F-layers, the reduction of $T_{\mathrm{c}}$ is caused by a SSV effect, generated by the singlet and the spin-projection zero triplet components, while at non-collinear alignment, especially in the crossed configuration (CR), it is caused by the triplet SSV effect. The obtained values are given in Figure $6 \mathrm{~b}$ as open circles. Since $T_{\mathrm{c}}$ in the APA state is smaller than predicted by the GL-like fit (corresponding to the positive or negative saturated (PS or NS) and, thus, parallel configuration), not a standard, but an inverse SSV effect [16] seems to be present. The maximum reduction in Figure $6 \mathrm{~b}$ is evaluated as $\Delta T_{\mathrm{c}, \max }$ and plotted in Figure 9a as a function of $d_{\mathrm{CuNi}}$.

Fominov et al. [15] suggested the descriptive explanation that the triplet component is generated from the singlet component in the vicinity of the $F_{1} / F_{2}$ interface, where the Cooper pairs "experience" an inhomogeneous magnetization. The amplitude of the singlet component in the $\mathrm{F}_{2}$ layer depends on the thickness of the $F_{1}$ layer, because the singlet component decays exponentially into the ferromagnetic material with a decay length of $2 l_{\mathrm{F} 1}$, where $l_{\mathrm{F} 1}$ is the electron mean free path in the material $F_{1}$. Here, we assume that the singlet wave function of the FFLO state in the $\mathrm{F}_{1}$ layer is best described by the extension of the dirty-limit theory towards the clean case, as obtained for $\mathrm{Nb} / \mathrm{Cu}_{41} \mathrm{Ni}_{59}$ bilayers $[8,9]$. In this case the decay length of the singlet state is given by $2 l_{\mathrm{F} 1}$ (see the Appendix of [54], in [9] the factor of 2 was omitted). However, the singlet $\mathrm{PWF}$ in the present $\mathrm{S} / \mathrm{F}_{1} / \mathrm{N} / \mathrm{F}_{2} / \mathrm{AF}$ heterostructure not only decays in the $\mathrm{F}_{1}$ layer, but also in the $\mathrm{N}$-layer, before reaching the $\mathrm{F}_{2}$ layer, i.e., it is reduced by an additional factor $\exp \left(-d_{\mathrm{N}} / \xi_{\mathrm{N}}\right)$. In our case, it is $d_{\mathrm{N}}=d_{\mathrm{nc}-\mathrm{Nb}} \approx 6 \mathrm{~nm}$ (see Figure 3). As discussed in the Appendix, $\xi_{\mathrm{N}}=20 \mathrm{~nm}$ is a suitable value. Thus, we assume that

$$
\Delta T_{\mathrm{c}, \max }\left(d_{\mathrm{CuNi}}, d_{\mathrm{N}}\right)=\Delta T_{\mathrm{c}, \max }\left(0, d_{\mathrm{N}}\right) \cdot e^{-\frac{d_{\mathrm{CuNi}}}{2 l_{\mathrm{F} 1}}}
$$

where $\Delta T_{\mathrm{c}, \max }\left(0, d_{\mathrm{N}}\right)=\Delta T_{\mathrm{c}, \max }(0,0) \cdot e^{-\left(d_{\mathrm{N}} / \xi_{\mathrm{N}}\right)}$. From the fit in Figure $9 \mathrm{a}$, we obtain $\Delta T_{\mathrm{c} \text {, max }}(0,6 \mathrm{~nm})=16.5 \mathrm{mK}$, yielding $\Delta T_{\mathrm{c}, \max }(0,0)=22.2 \mathrm{mK}$.

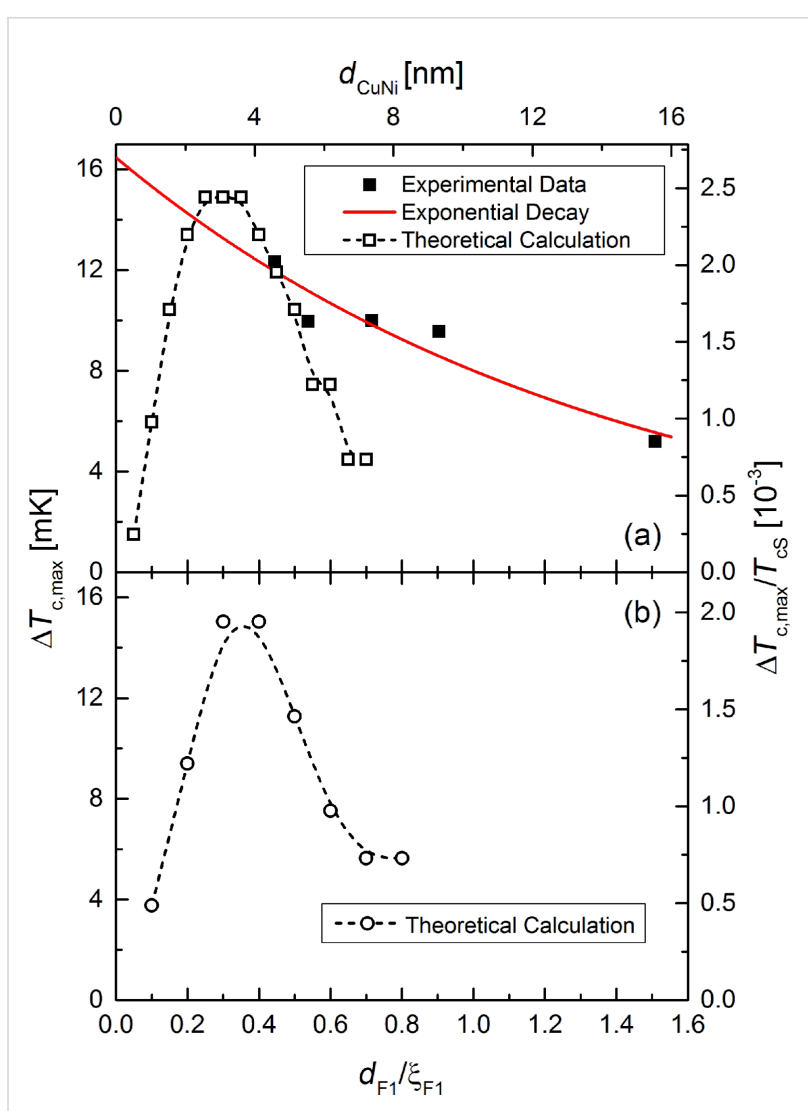

Figure 9: Maximum reduction of the transition temperature, $\Delta T_{\mathrm{c}, \max }$ by the triplet SSV effect at crossed configuration of the magnetic moments of the two ferromagnetic layers as a function of the thickness of the $\mathrm{Cu}_{41} \mathrm{Ni}_{59}$ layer. While the solid squares in (a) show experimental data, the open squares and circles in (a) and (b) show theoretical predictions as derived from the theory of Fominov and co-workers [15]. The theoretical data is plotted both in absolute and normalized (with respect to $T_{\mathrm{CS}}$ ) units. In (a), we assumed $T_{\mathrm{CS}}=6.1 \mathrm{~K}$, in (b) we used $T_{\mathrm{CS}}=7.7 \mathrm{~K}$, which is the critical temperature of a free standing $\mathrm{Nb}$ film of $12 \mathrm{~nm}$ thickness. The red line in (a) is a fit to an exponential decay according to Equation 3 , using $I_{\mathrm{F} 1}=7.1 \mathrm{~nm}$ and

$\Delta T_{\mathrm{c}, \max }(0.6 \mathrm{~nm})=16.5 \mathrm{mK}$. Moreover, we used $\xi_{\mathrm{F} 1}=10.3 \mathrm{~nm}$ for the magnetic coherence length in the $\mathrm{F}_{1}$ layer $\left(\mathrm{Cu}_{41} \mathrm{Ni}_{59}\right.$ layer) to plot the data as a function of the reduced thickness $d_{F 1} / \xi_{F 1}$. For a detailed discussion of the material and modeling parameters used for the theoretical calculations, see the Appendix.

The value of $l_{\mathrm{F} 1}=7.1 \mathrm{~nm}$ obtained from the fit is somewhat smaller than that in [9], where we obtained on average, that $l_{\mathrm{F} 1} / \xi_{\mathrm{F} 1}=1.18$ and $\xi_{\mathrm{F} 1}=10.3 \mathrm{~nm}$, so that $l_{\mathrm{F} 1}=12.2 \mathrm{~nm}$ (in that paper $l_{\mathrm{F} 1}$ and $\xi_{\mathrm{F} 1}$ are denoted by $l_{\mathrm{F}}$ and $\xi_{\mathrm{F} 0}$ ). This reduction of $l_{\mathrm{F} 1}$ may be the result of the formation of $\mathrm{NiO}$, which can serve as scattering centers, in the $F_{1}$ layer (see section Sample Preparation and Characterization). In our former work [72] electron mean free path values of $\mathrm{Cu}_{41} \mathrm{Ni}_{59}$ layers down to $5.9 \mathrm{~nm}$ were observed for samples probably aged under the influence of oxygen (see Table I of [72]).

From the theory of Fominov et al. [15] follows a breakdown of the triplet SSV effect for small $d_{\mathrm{F} 1}$, as shown in Figure 9. 
Apparently, for $d_{\mathrm{F} 1}=0$ the system becomes a $\mathrm{S} / \mathrm{N} / \mathrm{F}_{2}$ structure (possibly due to proximity-induced superconductivity even a $\mathrm{S} / \mathrm{F}_{2}$ bilayer) and, thus, the effect should be zero. The breakdown at small, but non-zero $d_{\mathrm{F} 1}$ can be explained by the fact, that if $d_{\mathrm{F} 1}$ becomes much smaller than the coherence length of the Cooper pairs in the $F_{1}$ material, they should not be able to "experience" the exchange interaction of the $\mathrm{F}_{1}$ layer. For large $d_{\mathrm{F} 1}$ the microscopic theory predicts a continuous decrease with increasing $d_{\mathrm{F} 1}$. Our measurements seem to show the decreasing tail for $d_{\mathrm{F} 1} / \xi_{\mathrm{F} 1}$ beyond the maximum of $\Delta T_{\mathrm{c}, \max }$.

There is a qualitative agreement between theory and experiment. In Figure 8 the predicted decrease of $T_{\mathrm{c} 0}\left(d_{\mathrm{F} 1}\right)$ is observed. However, in the experimentally investigated thickness range of $d_{\mathrm{F} 1}$ the calculated decrease is much to small. Moreover, the temperature ranges of $T_{\mathrm{c} 0}$ from experiment and theory only join, if $T_{\mathrm{cS}}=6.1 \mathrm{~K}$ is assumed. This is considerably lower than expected for an isolated $\mathrm{Nb}$ layer of $12 \mathrm{~nm}$ from our former investigations [9]. Naturally, we can not be sure, that the deposition conditions of the $\mathrm{Nb}$ layer in the present experiment are identical, so that a somewhat different critical temperature is possible. Especially the reactive deposition of $\mathrm{CoO}_{x}$ may decrease $T_{\mathrm{cS}}$ by decreasing the quality of the $\mathrm{Nb}$ film. A decreased critical temperature of the $\mathrm{Nb}$ film has also been assumed in our former work [72] to theoretically describe the experiments on samples, which were probably aged under the influence of oxygen (see Table I of [72], $d_{\mathrm{Nb}}$ is given in the figure captions, and Figure 5 of [9]).

In Figure 9, the decay of $\Delta T_{\mathrm{c} \text {,max }}\left(d_{\mathrm{F} 1}\right)$ predicted by the theory is much stronger than observed experimentally. Both in Figure 9a and Figure $9 \mathrm{~b}$, the predicted absolute range of $\Delta T_{\mathrm{c}, \max }$ fit the experimental data. One possible reason for the observed deviations between the theoretical predictions and the experimental data is that the theory of Fominov et al. [15] is only valid for dirty ferromagnetic material (i.e., if $l_{F 1} \ll \xi_{F 1}$ ). However, neither the $\mathrm{Cu}_{41} \mathrm{Ni}_{59}$ layer nor the Co layer are in the dirty limit. While for Co this is obvious, we concluded in our former work [9], that $\mathrm{Cu}_{41} \mathrm{Ni}_{59}$ is between the dirty and clean limit (i.e., if $\left.l_{F 1} \gg \xi_{F 1}\right)$. Moreover, we also observed that the oscillations in $T_{\mathrm{c}}\left(d_{\mathrm{F}}\right)$ are better described by an extension of the dirty-case theory towards the clean limit than by the dirty theory $[8,9]$.

Moreover, the reduced atomic magnetic moment for $\mathrm{Cu}_{41} \mathrm{Ni}_{59}$ in our samples probably leads to a decrease of $E_{\mathrm{ex}}$ and, thus, an increase of $\xi_{\mathrm{F} 1}$ beyond $10.3 \mathrm{~nm}$ found in investigations of S/Fbilayers [9]. In more recent investigations [72], values up to $17 \mathrm{~nm}$ are observed for $\mathrm{Cu}_{41} \mathrm{Ni}_{59}$ films, which were aged or exposed to an oxygen atmosphere after deposition of the $\mathrm{Cu}_{41} \mathrm{Ni}_{59}$ film. Using such a value to scale the experimental data, would yield the data point with the largest thickness of
$d_{\mathrm{CuNi}} \approx 15 \mathrm{~nm}$ to correspond to a reduced thickness of $d_{\mathrm{F} 1} / \xi_{\mathrm{F} 1}=0.9$, narrowing the gap between theory and experiment. However, since $\xi_{\mathrm{F} 1}$ is connected to $E_{\mathrm{ex}}$, which influences further parameters of the calculation, for a final statement detailed theoretical calculations with increased values of $\xi_{\mathrm{F} 1}$ and decreased $E_{\text {ex }}$ need to be carried out.

Basically, the observed triplet SSV effect may be masked by the generation of a spin-projection one triplet component at domain walls (where the magnetization is also non-uniform) [49]. The occurrence of domain walls is expected just at the same applied field as the triplet SSV effect, i.e., at the coercive field [65]. This effect is not considered in the theory of Fominov and co-workers [15]. However, the multi-domain state should also be present in $\mathrm{S} / \mathrm{F}$-bilayers of $\mathrm{Nb} / \mathrm{Cu}_{41} \mathrm{Ni}_{59}$ at the coercive field, at which no reduction of $T_{\mathrm{c}}$ was observed in our former work (see the discussion at the end of Chapter B.2 in [73]).

\section{Conclusion}

In the present work we investigated the triplet superconducting spin-valve (SSV) effect in a $\mathrm{S} / \mathrm{F}_{1} / \mathrm{N} / \mathrm{F}_{2} / \mathrm{AF}$ heterostructure with $\mathrm{S}=\mathrm{Nb} ; \mathrm{F}_{1}=\mathrm{Cu}_{41} \mathrm{Ni}_{59} ; \mathrm{N}=\mathrm{nc}-\mathrm{Nb} ; \mathrm{F}_{2}=\mathrm{Co} ; \mathrm{AF}=\mathrm{CoO}_{x}$. The experiments show a decay of the effect with increasing thickness of the $\mathrm{F}_{1}$ layer, $d_{\mathrm{F} 1}$.

The microscopic theory predicts a breakdown of the effect for very thin $\mathrm{F}_{1}$ layers. Apparently, for $d_{\mathrm{F} 1}=0$ the system becomes a $\mathrm{S} / \mathrm{N} / \mathrm{F}_{2}$ structure (possibly due to proximity-induced superconductivity even a $S / F_{2}$ bilayer) and, thus, the effect should be zero. The breakdown at small, but non-zero $d_{\mathrm{F} 1}$ can be explained by the fact, that if $d_{\mathrm{F} 1}$ becomes much smaller than the coherence length of the Cooper pairs in the $F_{1}$ material, they should not be able to "experience" the exchange interaction of the $\mathrm{F}_{1}$ layer. For large $d_{\mathrm{F} 1}$ the microscopic theory predicts a continuous decrease with increasing $d_{\mathrm{F} 1}$, as observed in the present work.

We modeled the experimental data both, with the microscopic theory, as well as an empirical model, which predicts an exponential decay with the decay length of $2 l_{\mathrm{F} 1}$, where $l_{\mathrm{F} 1}$ is the electron mean free path in the $F_{1}$ material. This model is based on a simple picture, in which the reason for the decay of the triplet SSV effect with increasing $d_{\mathrm{F} 1}$ is the exponential spatial decay of the singlet FFLO pairing wave function in the $\mathrm{F}_{1}$ layer. From this wave function the triplet component is generated mainly at the border of the $\mathrm{F}_{2}$ material.

The empirical model yields a quantitative agreement with the data with $l_{\mathrm{F} 1}$ of the order of the electronic mean free path in $\mathrm{Cu}_{41} \mathrm{Ni}_{59}$. The decay of the effect with increasing $d_{\mathrm{F} 1}$ is, however, only in qualitative agreement with the microscopic theory. 
For quantitative agreement between microscopic theory and experiment (if possible at all for clean, e.g., Co, or intermediate, e.g., $\mathrm{Cu}_{41} \mathrm{Ni}_{59}$, ferromagnets), a more detailed theoretical study of the influence of the coherence length and the exchange energy of the $F_{1}$ material has to be performed.

\section{Appendix \\ Material parameters of the theoretical calculations in Figure $8 b$ and Figure 9b}

The critical temperature of a free standing $\mathrm{Nb}$ film of $12 \mathrm{~nm}$, as in our heterostructure, is $7.7 \mathrm{~K}$ according to Figure 5 of [9]. The coherence length $\xi_{S}=6.68 \mathrm{~nm}$ [9] for a free standing $\mathrm{Nb}$ film of $14 \mathrm{~nm}$ thickness, which is close to $d_{\mathrm{S}}$ of the samples in the present work. Moreover, $\xi_{\mathrm{F} 1}=10.3 \mathrm{~nm}$ (average of the values $\xi_{\mathrm{F} 0}$ given in [9]). For $\mathrm{F}_{2}=$ Co we take $\xi_{\mathrm{F} 2}=1.3 \mathrm{~nm}$ from [56] and $d_{\mathrm{F} 2} / \xi_{\mathrm{F} 2}=15$, i.e., $d_{\mathrm{F} 2}=19.5 \mathrm{~nm}$, which is close to the midpoint of $d_{\text {Co }}$ of the investigated samples (see Figure 3).

For the N spacer, the decay length of the PWF is given by [75] $\xi_{\mathrm{N}}=\left(\hbar D /\left(2 \pi k_{\mathrm{B}} T\right)\right)^{1 / 2}$, where $D=(1 / 3) l_{\mathrm{N}} v_{\mathrm{F}}$ is the diffusion constant. Here, $l_{\mathrm{N}}$ is the electron mean free path, and $v_{\mathrm{F}}$ is the Fermi velocity, $\hbar=h / 2 \pi$ with $h$ being Planck's constant, and $k_{\mathrm{B}}$ the Boltzmann constant. As our $\mathrm{N}$ layer is nc-Nb, we take $v_{\mathrm{F}}=2.768 \times 10^{5} \mathrm{~m} / \mathrm{s}$, according to [76], and $l_{\mathrm{N}}=2.1 \mathrm{~nm}$, as determined for a $7.3 \mathrm{~nm}$ thick $\mathrm{Nb}$ film (which is close to the thickness, $d_{\mathrm{nc}-\mathrm{Nb}} \approx 6 \mathrm{~nm}$, of the nc-Nb spacer in our heterostructure) in Appendix B.4 of [73]. For $T$, we insert $3.6 \mathrm{~K}$, which is the midpoint temperature of the temperature range of the $T_{\mathrm{c} 0}$ values in Figure 8a.

For the exchange splitting energy of the $\mathrm{Cu}_{41} \mathrm{Ni}_{59}$ layer we take $E_{\text {ex }, \mathrm{CuNi}}=14.7 \mathrm{meV}$, as obtained in [77] for $\mathrm{Cu}_{40} \mathrm{Ni}_{60}$ films. As $1 \mathrm{eV}=k_{\mathrm{B}} \cdot 11604 \mathrm{~K}$ and $T_{\mathrm{cS}}=7.7 \mathrm{~K}$, we get $h_{\mathrm{F} 1}=E_{\mathrm{Ex}, \mathrm{CuNi}} /\left(\pi k_{\mathrm{B}} T_{\mathrm{cS}}\right)=7.0$. For Co $E_{\mathrm{Ex}, \mathrm{Co}}=0.93-1.05 \mathrm{eV}$ according to [78]. Taking $E_{\mathrm{Ex}, \mathrm{Co}}=1.0 \mathrm{eV}$ we get $h_{\mathrm{F} 2}=E_{\mathrm{Ex}, \mathrm{Co}} /\left(\pi k_{\mathrm{B}} T_{\mathrm{cS}}\right)=480$.

Furthermore, we used the following proximity strength parameters as modeling parameters: $\gamma_{\mathrm{F} 1, \mathrm{~S}}=0.2, \gamma_{\mathrm{N}, \mathrm{F} 1}=1.0, \gamma_{\mathrm{F} 2, \mathrm{~N}}=10$, $\gamma_{\mathrm{B}, \mathrm{F} 1, \mathrm{~S}}=0.5, \gamma_{\mathrm{B}, \mathrm{N}, \mathrm{F} 1}=0.2, \gamma_{\mathrm{B}, \mathrm{F} 2, \mathrm{~N}}=3$ (see definitions in [79]).

\section{Material parameters of the theoretical calculations in Figure 8a and Figure 9a}

Unless otherwise stated, the material parameters are the same as mentioned in the first section of the Appendix. The critical temperature of the free standing $\mathrm{Nb}$ film is assumed to be $T_{\mathrm{cS}}=6.1 \mathrm{~K}$, yielding $h_{\mathrm{F} 1}=8.8$ and $h_{\mathrm{F} 2}=606$. If we take into account an enhancement factor of the slope of the temperature dependence of the upper critical field in [9], we obtain the coherence length in the superconductor $\xi_{S}=7.3 \mathrm{~nm}$. According to Butler [80], this factor is 1.18 for $\mathrm{Nb}$ in the dirty limit.

\section{Acknowledgements}

The authors are grateful to S. Heidemeyer, B. Knoblich, and W. Reiber for the TEM-sample preparation, additionally to W. Reiber for assistance in RBS measurements, and to D. Vieweg for assistance in magnetic measurements. This work was supported by the Deutsche Forschungsgemeinschaft (DFG) under the Grant No. HO 955/9-1. The partial support by RFBR (grants Nos 14-02-00793-a, L.R.T. and R.G.D.; 15-52-10045KO-a, M.Yu.K.), STCU (grant No. 5982, A.S.S. and R.M.), and by the Program of Competitive Growth of Kazan Federal University funded by the Russian Government (R.M., M.Yu.K., and L.R.T.) is acknowledged. V.I.Z. was partially supported by ERC advanced grant "ASTONISH". The magnetic investigations (H.-A. K.v.N.) were partially supported by the Deutsche Forschungsgemeinschaft (DFG) within the Transregional Collaborative Research Center TRR 80 "From Electronics Correlations to Functionality" (Augsburg-Munich).

\section{References}

1. Fulde, P.; Ferrell, R. A. Phys. Rev. 1964, 135, A550. doi:10.1103/PhysRev.135.A550

2. Larkin, A. I.; Ovchinnikov, Y. N. Sov. Phys. - JETP 1965, 20, 762.

3. Bardeen, J.; Cooper, L. N.; Schrieffer, J. R. Phys. Rev. 1957, 108, 1175. doi:10.1103/PhysRev.108.1175

4. Zwicknagl, G.; Wosnitza, J. Int. J. Mod. Phys. B 2010, 24, 3915. doi:10.1142/S0217979210056396

5. Buzdin, A. I. Rev. Mod. Phys. 2005, 77, 935. doi:10.1103/RevModPhys.77.935

6. Eschrig, M. Phys. Today 2011, 64, 43. doi:10.1063/1.3541944

7. Tagirov, L. R. Physica C 1998, 307, 145. doi:10.1016/S0921-4534(98)00389-X

8. Zdravkov, V.; Sidorenko, A.; Obermeier, G.; Gsell, S.; Schreck, M.; Müller, C.; Horn, S.; Tidecks, R.; Tagirov, L. R. Phys. Rev. Lett. 2006, 97, 057004. doi:10.1103/PhysRevLett.97.057004

9. Zdravkov, V. I.; Kehrle, J.; Obermeier, G.; Gsell, S.; Schreck, M.; Müller, C.; Krug von Nidda, H.-A.; Lindner, J.; Moosburger-Will, J.; Nold, E.; Morari, R.; Ryazanov, V. V.; Sidorenko, A. S.; Horn, S.; Tidecks, R.; Tagirov, L. R. Phys. Rev. B 2010, 82, 054517. doi:10.1103/PhysRevB.82.054517

10. Oh, S.; Youm, D.; Beasley, M. R. Appl. Phys. Lett. 1997, 71, 2376. doi:10.1063/1.120032

11. Tagirov, L. R. Phys. Rev. Lett. 1999, 83, 2058. doi:10.1103/PhysRevLett.83.2058

12. Buzdin, A. I.; Vedyayev, A. V.; Ryzhanova, N. V. Europhys. Lett. 1999, 48, 686. doi:10.1209/epl/i1999-00539-0

13. You, C.-Y.; Bazaly, Ya. B.; Gu, J. Y.; Oh, S.-J.; Litvak, L. M.; Bader, S. D. Phys. Rev. B 2004, 70, 014505. doi:10.1103/PhysRevB.70.014505

14. Fominov, Ya. V.; Golubov, A. A.; Kupriyanov, M. Yu. JETP Lett. 2003, 77, 510. doi:10.1134/1.1591981

15. Fominov, Ya. V.; Golubov, A. A.; Karminskaya, T. Yu.; Kupriyanov, M. Yu.; Deminov, R. G.; Tagirov, L. R. JETP Lett. 2010, 91, 308. doi:10.1134/S002136401006010X

16. Mironov, S. V.; Buzdin, A. Phys. Rev. B 2014, 89, 144505. doi:10.1103/PhysRevB.89.144505 
17. Gu, J. Y.; You, C.-Y.; Jiang, J. S.; Pearson, J.; Bazaliy, Ya. B.; Bader, S. D. Phys. Rev. Lett. 2002, 89, 267001. doi:10.1103/PhysRevLett.89.267001

18. Potenza, A.; Marrows, C. H. Phys. Rev. B 2005, 71, 180503. doi:10.1103/PhysRevB.71.180503

19. Gu, J. Y.; Bazaliy, Ya. B.; Bader, S. D.; You, C. Y. Ferromagnetic-Superconducting Hybrid Systems. In Theory of Quantum Transport in Metallic and Hybrid Nanostructures; Glatz, A.; Kozub, V. I.; Vinokur, V. M., Eds.; NATO Science Series, Vol. 230; Springer: Dordrecht, Netherlands, 2006; pp 159-171. doi:10.1007/1-4020-4779-7_15

20. Moraru, I. C.; Pratt, W. P., Jr.; Birge, N. O. Phys. Rev. Lett. 2006, 96, 037004. doi:10.1103/PhysRevLett.96.037004

21. Moraru, I. C.; Pratt, W. P., Jr.; Birge, N. O. Phys. Rev. B 2006, 74, 220507. doi:10.1103/PhysRevB.74.220507

22. Rusanov, A. Yu.; Habraken, S.; Aarts, J. Phys. Rev. B 2006, 73 , 060505. doi:10.1103/PhysRevB.73.060505

23. Aarts, J.; Rusanov, A. Yu. C. R. Phys. 2006, 7, 99. doi:10.1016/j.crhy.2005.12.005

24. Steiner, R.; Ziemann, P. Phys. Rev. B 2006, 74, 094504 doi:10.1103/PhysRevB.74.094504

25. Stamopoulos, D.; Manios, E.; Pissas, M. Phys. Rev. B 2007, 75, 014501. doi:10.1103/PhysRevB.75.014501

26. Singh, A.; Sürgers, C.; v. Löhneysen, H. Phys. Rev. B 2007, 75, 024513. doi:10.1103/PhysRevB.75.024513

27. Singh, A.; Sürgers, C.; Hoffmann, R.; v. Löhneysen, H.; Ashworth, T. V.; Pilet, N.; Hug, H. J. Appl. Phys. Lett. 2007, 91, 152504. doi:10.1063/1.2794424

28. Kim, D. H.; Hwang, T. J. Physica C 2007, 455, 58. doi:10.1016/j.physc.2007.02.007

29. Nowak, G.; Zabel, H.; Westerholt, K.; Garifullin, I.; Marcellini, M.; Liebig, A.; Hjörvarsson, B. Phys. Rev. B 2008, 78, 134520. doi:10.1103/PhysRevB.78.134520

30. Cadden-Zimansky, P.; Bazaliy, Ya. B.; Litvak, L. M.; Jiang, J. S.; Pearson, J.; Gu, J. Y.; You, C.-Y.; Beasley, M. R.; Bader, S. D. Phys. Rev. B 2008, 77, 184501. doi:10.1103/PhysRevB.77.184501

31. Leksin, P. V.; Salikhov, R. I.; Garifullin, I. R.; Vinzelberg, H.; Kataev, V.; Klingeler, R.; Tagirov, L. R.; Büchner, B. JETP Lett. 2009, 90, 59. doi:10.1134/S0021364009130128

32. Zhu, J.; Cheng, X.; Boone, C.; Krivorotov, I. N. Phys. Rev. Lett. 2009, 103, 027004. doi:10.1103/PhysRevLett.103.027004

33. Zhu, J.; Krivorotov, I. N.; Halterman, K.; Valls, O. T. Phys. Rev. Lett. 2010, 105, 207002. doi:10.1103/PhysRevLett.105.207002

34. Luo, Y.; Samwer, K. Europhys. Lett. 2010, 91, 37003. doi:10.1209/0295-5075/91/37003

35. Gu, Y.; Halász, G. B.; Robinson, J. W. A.; Blamire, M. G. Phys. Rev. Lett. 2015, 115, 067201. doi:10.1103/PhysRevLett.115.067201

36. Leksin, P. V.; Garif'yanov, N. N.; Garifullin, I. A.; Schumann, J.; Vinzelberg, H.; Kataev, V.; Klingeler, R.; Schmidt, O. G.; Büchner, B. Appl. Phys. Lett. 2010, 97, 102505. doi:10.1063/1.3486687

37. Leksin, P. V.; Garif'yanov, N. N.; Garifullin, I. A.; Schumann, J.; Kataev, V.; Schmidt, O. G.; Büchner, B. Phys. Rev. Lett. 2011, 106, 067005. doi:10.1103/PhysRevLett.106.067005

38. Leksin, P. V.; Garif'yanov, N. N.; Garifullin, I. A.; Schumann, J.; Kataev, V.; Schmidt, O. G.; Büchner, B. Phys. Rev. B 2012, 85 , 024502. doi:10.1103/PhysRevB.85.024502

39. Nowak, G.; Westerholt, K.; Zabel, H. Supercond. Sci. Technol. 2013, 26, 025004. doi:10.1088/0953-2048/26/2/025004
40. Leksin, P. V.; Kamashev, A. A.; Garif'yanov, N. N.; Garifullin, I. A.; Fominov, Ya. V.; Schumann, J.; Hess, C.; Kataev, V.; Büchner, B. JETP Lett. 2013, 97, 478. doi:10.1134/S0021364013080109

41. Zdravkov, V. I.; Kehrle, J.; Obermeier, G.; Lenk, D.; Krug von Nidda, H.-A.; Müller, C.; Kupriyanov, M. Yu.; Sidorenko, A. S.; Horn, S.; Tidecks, R.; Tagirov, L. R. Phys. Rev. B 2013, 87, 144507. doi:10.1103/PhysRevB.87.144507

42. Leksin, P. V.; Garif'yanov, N. N.; Garifullin, I. A.; Fominov, Ya. V.; Schumann, J.; Krupskaya, Y.; Kataev, V.; Schmidt, O. G.; Büchner, B. Phys. Rev. Lett. 2012, 109, 057005. doi:10.1103/PhysRevLett.109.057005

43. Wang, X. L.; Di Bernado, A.; Banerjee, N.; Wells, A.; Bergeret, F. S.; Blamire, M. G.; Robinson, J. W. A. Phys. Rev. B 2014, 89, 140508. doi:10.1103/PhysRevB.89.140508

44. Jara, A. A.; Safranski, C.; Krivorotov, I. N.; Wu, C.-T.; Malmi-Kakkada, A. N.; Valls, O.; Halterman, K. Phys. Rev. B 2014, 89, 184502. doi:10.1103/PhysRevB.89.184502

45. Singh, A.; Voltan, S.; Lahabi, K.; Aarts, J. Phys. Rev. X 2015, 5, 021019. doi:10.1103/PhysRevX.5.021019

46. Flokstra, M. G.; Cunningham, T. C.; Kim, J.; Satchell, N.; Burnell, G.; Curran, P. J.; Bending, S. J.; Kinane, C. J.; Cooper, J. F. K.; Langridge, S.; Isidori, A.; Pugach, N.; Eschrig, M.; Lee, S. L. Phys. Rev. B 2015, 91, 060501. doi:10.1103/PhysRevB.91.060501

47. Leksin, P. V.; Garif'yanov, N. N.; Kamashev, A. A.; Fominov, Ya. V.; Schumann, J.; Hess, C.; Kataev, V.; Büchner, B.; Garifullin, I. A. Phys. Rev. B 2015, 91, 214508. doi:10.1103/PhysRevB.91.214508

48. Banerjee, N.; Smiet, C. B.; Smits, R. G. J.; Ozaeta, A.; Bergeret, F. S.; Blamiere, M. G.; Robinson, J. W. A. Nat. Commun. 2014, 5, 3048. doi:10.1038/ncomms4048

49. Bergeret, F. S.; Volkov, A. F.; Efetov, K. B. Rev. Mod. Phys. 2005, 77, 1321. doi:10.1103/RevModPhys.77.1321

50. Bergeret, F. S.; Volkov, A. F.; Efetov, K. B. Phys. Rev. Lett. 2001, 86, 4096. doi:10.1103/PhysRevLett.86.4096

51. Volkov, A. F.; Bergeret, F. S.; Efetov, K. B. Phys. Rev. Lett. 2003, 90, 117006. doi:10.1103/PhysRevLett.90.117006

52. Khaire, T. S.; Khasawneh, M. A.; Pratt, W. P., Jr.; Birge, N. O. Phys. Rev. Lett. 2010, 104, 137002. doi:10.1103/PhysRevLett.104.137002

53. Ruotolo, A.; Bell, C.; Leung, C. W.; Blamire, M. G. J. Appl. Phys. 2004, 96, 512. doi:10.1063/1.1757658

54. Kehrle, J.-M. The Fulde-Ferrell Larkin-Ovchinnikov Like State in Bilayers and Trilayers of Superconducting and Ferromagnetic Thin Films. Ph.D. Thesis, Universität Augsburg, Augsburg, Germany, 2012.

55. Radu, F.; Zabel, H. Exchange Bias Effect of Ferro-/Antiferromagnetic Heterostructures. In Magnetic Heterostructures; Zabel, H.; Bader, S. D., Eds.; Springer Tracts in Modern Physics, Vol. 227; Springer: Berlin, Germany, 2008; pp 97-184.

56. Liang, J.-J.; Lee, S. F.; Shih, W. T.; Chang, W. L.; Yu, C.; Yao, Y. D. J. Appl. Phys. 2002, 92, 2624. doi:10.1063/1.1496126

57. Sidorenko, A. S.; Zdravkov, V. I.; Prepelitsa, A. A.; Helbig, C.; Luo, Y.; Gsell, S.; Schreck, M.; Klimm, S.; Horn, S.; Tagirov, L. R.; Tidecks, R. Ann. Phys. 2003, 12, 37. doi:10.1002/andp.200310005

58. Zdravkov, V. I.; Kehrle, J.; Obermeier, G.; Ullrich, A.; Gsell, S.; Lenk, D.; Müller, C.; Morari, R.; Sidorenko, A. S.; Ryazanov, V. V.; Tagirov, L. R.; Tidecks, R.; Horn, S. Supercond. Sci. Technol. 2011, 24, 095004. doi:10.1088/0953-2048/24/9/095004

59. Lindner, J.; Robinson, J. W. A. Nat. Phys. 2015, 11, 307. doi:10.1038/nphys3242 
60. Flokstra, M. G.; Satchell, N.; Kim, J.; Burnell, G.; Curran, P. J.; Bending, S. J.; Cooper, J. F. K.; Kinane, C. J.; Langridge, S.; Isidori, A.; Pugach, N.; Eschrig, M.; Luetkens, H.; Suter, A.; Prokscha, T.; Lee, S. L. Nat. Phys. 2015, 12, 57-61. doi:10.1038/nphys3486 61. Robinson, J. Physics 2015, 8, 49.

62. Geiler, A. L.; Harris, V. G.; Vittoria, V.; Sun, N. X. J. Appl. Phys. 2006, 99, 08B316. doi:10.1063/1.2170061

63. Goldfarb, R. B.; Fickett, F. R. NBS Special Publication No. 696; National Bureau of Standards: Boulder, CO, U.S.A., 1985. For sale by the Superintendent of Documents U.S. Government Printing Office, Washington, DC 20402.

64. Chikazumi, S. Physics of Ferromagnetism, 2nd ed.; Oxford University Press: Oxford, United Kingdom, 1985; §19.1.

65. Coey, J. M. P. Magnetism and Magnetic Materials; Cambridge University Press: Cambridge, United Kingdom, 2010.

66. Murthy, V. S. R.; Jena, A. K.; Gupta, K. P.; Murthy, G. S. Magnetic Properties of Materials. Structure and Properties of Engineering Materials; Tata McGraw-Hill: New Delhi, India, 2003; p 381.

67. Ahern, S. A.; Martin, M. J. C.; Sucksmith, W. Proc. R. Soc. London 1958, A248, 145. doi:10.1098/rspa.1958.0235

68. Fritzsche, H.; Kohlhepp, J.; Gradmann, U. Phys. Rev. B 1995, 51, 15933. doi:10.1103/PhysRevB.51.15933

69. Massalski, T. B.; Okamoto, H.; Subramaniam, P. R.; Kacprzak, L. Binary Alloy Phase Diagrams, 2nd ed.; ASM International: Materials Park, OH, U.S.A., 1990.

70. Garcia, M. A.; Fernandez-Pinel, E.; de la Venta, J.; Quesada, A.; Bouzas, V.; Fernández, J. F.; Romero, J. J.; Martín-González, M. S.; Costa-Krämer, J. L. J. Appl. Phys. 2009, 105, 013925. doi:10.1063/1.3060808

71. Hautot, D.; Pankhurst, Q. A.; Dobsen, J. Rev. Sci. Instrum. 2005, 76, 045101. doi:10.1063/1.1868272

72. Zdravkov, V. I.; Kehrle, J.; Lenk, D.; Obermeier, G.; Ullrich, A.; Müller, C.; Krug von Nidda, H. A.; Morari, R.; Sidorenko, A. S.; Tagirov, L. R.; Horn, S.; Tidecks, R. J. Appl. Phys. 2013, 114, 033903. doi:10.1063/1.4813131

73. Lenk, D.; Hemmida, M.; Morari, R.; Zdravkov, V. I.; Ullrich, A.; Müller, C.; Sidorenko, A. S.; Horn, S.; Tagirov, L. R.; Loidl, A.; Krug von Nidda, H.-A.; Tidecks, R. Phys. Rev. B 2016, 93, 184501. doi:10.1103/PhysRevB.93.184501

74. Tinkham, M. Introduction to Superconductivity, 2nd ed.; McGraw Hill: New York, NY, U.S.A., 1996.

75. Deutscher, G.; de Gennes, P. G. Proximity Effects. In Superconductivity; Parks, R. D., Ed.; Marcel Dekker: New York, NY, U.S.A., 1996; Vol. 2.

76. Weber, H. W.; Seidl, E.; Laa, C.; Schachinger, E.; Prohammer, M.; Junod, A.; Eckert, D. Phys. Rev. B 1991, 44, 7585. doi:10.1103/PhysRevB.44.7585

77. Kim, J.; Kwon, J. H.; Char, K.; Doh, H.; Choi, H.-Y. Phys. Rev. B 2005, 72, 014518. doi:10.1103/PhysRevB.72.014518

78. Himpsel, F. J.; Altmann, K. N.; Mankey, G. J.; Ortega, J. E.; Petrovykh, D. Y. J. Magn. Magn. Mater. 1999, 200, 456. doi:10.1016/S0304-8853(99)00349-2

79. Aarts, J.; Geers, J. M. E.; Brück, E.; Golubov, A. A.; Coehoorn, R. Phys. Rev. B 1997, 56, 2779. doi:10.1103/PhysRevB.56.2779

80. Butler, W. H. Phys. Rev. Lett. 1980, 44, 1516. doi:10.1103/PhysRevLett.44.1516

\section{License and Terms}

This is an Open Access article under the terms of the Creative Commons Attribution License

(http://creativecommons.org/licenses/by/2.0), which permits unrestricted use, distribution, and reproduction in any medium, provided the original work is properly cited.

The license is subject to the Beilstein Journal of Nanotechnology terms and conditions: (http://www.beilstein-journals.org/bjnano)

The definitive version of this article is the electronic one which can be found at: doi:10.3762/bjnano.7.88 